\title{
Zur Einführung: Recht und Sozialwissenschaften - Herausforderungen und Chancen der Verhaltensökonomie
}

I.

Heft 1-2 des Jahrgangs 2007 der KritV dokumentiert die Werkstattgespräche zum Thema »Uncertainty, Risk and Regulation: The Behavioral Law and Economics Perspective «, zu denen sich die Teilnehmer am 16. und 17. Juni 2006 in der TU Berlin getroffen haben ${ }^{1}$. Zweck der Veranstaltung war, einige für Rechtswissenschaft und/oder Rechtspraxis relevante Forschungsprojekte aus dem Bereich von behavioral economics vorzustellen und im Kreise einschlägig interessierter Ökonomen und Juristen zu diskutieren.

Hintergrund der damit verfolgten Absichten ist der Befund, dass die Verwendung und Verwertung wirtschaftswissenschaftlicher Methoden und Einsichten in der deutschen Jurisprudenz nach wie vor unterentwickelt ist. ${ }^{2}$ Zwar ist die ursprünglich nicht seltene offene Ablehnung ${ }^{3}$ zur Ausnahme geworden ${ }^{4}$. Sie ist angesichts fundierter rechtsmethodischer Begründungen ${ }^{5}$ auch sehr viel schwieriger geworden. Ein höchst verdienstliches Lehrbuch hat sich etabliert ${ }^{6}$. Und eine wachsende Zahl sachlich informierter und methodisch umsichtiger Abhandlungen - etwa zum Deliktsrecht ${ }^{7}$, zum Vertragsrecht ${ }^{8}$ oder zum Gesellschafts- und Kapitalmarktrecht ${ }^{9}$ - verdeutlicht die Chancen, die die Kooperation mit den Wirtschaftswissenschaften nicht nur der Theorie, sondern auch der Rechtspraxis zu eröffnen vermag.

Von einem durchgreifenden Wandel kann aber nach wie vor nicht die Rede sein. Die erwähnten Publikationen sind weiterhin ganz kleine Inseln in einem Ozean ausschließlich dogmatischer Literatur. Ihre praktische Bedeutung bleibt gering: Es ist nicht ersichtlich, dass ihre Einsichten und Empfehlungen von der Rechtsprechung übernommen werden ${ }^{10}$. Auch der Einfluss auf die Gesetzgebung ist minimal geblie-

1 Der Workshop wurde organisiert von Prof. Dr. Dominique Demougin, HU Berlin, und Prof. Dr. Dorothea Kübler, TU Berlin; er wurde von der DFG finanziert aus Mitteln des SFB $649 »$ Economic Risk «.

2 Dau-Schmidt/Brun, Lost in Translation: The Economic Analysis of Law in the United States and Europe, Columbia Journal of Transnational Law 44, 602 ff. (2006).

3 Fezer JZ 1986, 817 ff.; Eder, Z. f. Rechtssoz. 7 (1986), 1 ff.; Bydlinski AcP 188 (1988), $447,460$.

4 Vgl. aber neuerdings wieder Rittner JZ 2005, 668, 669.

5 Eidenmüller, Effizienz als Rechtsprinzip (2. Aufl. 1998); s. auch schon Behrens, Politische Ökonomie als rationale Jurisprudenz (1986).

6 Schäfer/Ott, Lehrbuch der ökonomischen Analyse des Zivilrechts (4. Aufl. 2004).

7 Taupitz AcP 1996 (1996), 110 ff.

8 Eidenmüller JZ 2005, $216 \mathrm{ff}$.

9 Fleischer ZGR 2001, 1 ff.

10 Vgl. Taupitz S. 120: »Es gibt, soweit ersichtlich, keine einzige gerichtliche Entscheidung, in der das Ergebnis ausdrücklich auf die ökonomische Analyse gestützt worden wäre«. 
ben. Und in der Ausbildung der nachwachsenden Juristen sind keine Änderungen ersichtlich: An allen Fakultäten gibt es Professuren für Rechtsgeschichte, für Rechtssoziologie, für allgemeine Staatslehre und Kriminologie, für Rechtstheorie und Rechtsphilosophie, aber - von ganz wenigen Ausnahmen abgesehen - keine entsprechenden Positionen für die Rechtsökonomie. Deshalb gibt es keine einschlägigen Lehrveranstaltungen. Für die meisten Studierenden bleiben die mikroökonomischen Implikationen des Rechts und seiner Durchsetzung ein völlig unbekanntes Terrain.

Das unterscheidet die deutsche Situation nicht nur von der in den USA ${ }^{11}$. Auch in anderen - europäischen und nichteuropäischen Ländern - wird der Rechtsökonomie wachsende Bedeutung zugemessen. Das konfrontiert mit der Frage, welche Kosten die deutsche Abstinenz nach sich zieht. Gibt es Grund zu der Annahme, dass der offensichtliche Reformstau in einer breit verstandenen Wirtschaftsrechtspolitik gewiss nicht allein aber doch auch darauf beruht, dass die maßgeblichen Akteure nicht gelernt haben und nicht bereit sind, die anstehenden Probleme auch als ökonomische Herausforderungen zu begreifen? Und die Praxis bedient sich zunehmend auf anderen Märkten: Die großen Anwaltssozietäten, die vorwiegend wirtschaftsrechtliche Beratung anbieten, stellen kaum noch einen Nachwuchsjuristen ein, der nicht durch den Erwerb eines amerikanischen oder britischen LLM den Nachweis erbringt, dass er Lehrveranstaltungen absolviert hat, in denen die Probleme etwa des Gesellschafts- oder Insolvenzrechts auch im Lichte ökonomischer Fragestellungen untersucht und erörtert werden. Das heißt: Für diese Form der Rechtspraxis gilt das deutsche System der Ausbildung als nicht mehr zureichend.

\section{II.}

Die deutsche Verspätung hat institutionelle Gründe, die überwiegend auf den sklerotisierten Strukturen der Ausbildung beruhen. Der der Tradition verhaftete Staatsexamensbetrieb erschwert die Anpassung des Lehrprogramms an sich wandelnde Bedürfnisse; er bietet den Studierenden keine Anreize, sich interdisziplinär zu betätigen. Das starre Festhalten an den überlieferten Fachgrenzen verhindert, dass Wirtschaftswissenschaftler in juristische Fakultäten berufen und dort mit kartell- oder kapitalmarktrechtlichen Lehrveranstaltungen betraut werden - ein in den USA nicht ungewöhnlicher Vorgang. Das schlägt auf die Motivation des wissenschaftlichen Nachwuchses zurück: Rechtsökonomische Forschung erscheint wenig lohnend; sie ist mühsam und nicht geeignet, die Berufungschancen zu fördern.

Nicht weniger wichtig erscheinen die wissenschaftlichen Vorbehalte, die gegen die Rechtsökonomie geltend gemacht werden. Es sind vor allem zwei Argumente, die immer wieder vorgetragen werden ${ }^{12}$. Zunächst wird der Verlust der Eigenständigkeit des Rechts bzw. der Rechtswissenschaft beschworen. Beide lieferten sich einer fremden Disziplin, den Wirtschaftswissenschaften, aus ${ }^{13}$. Dieser Einwand geht von einem sehr

11 Dazu Taupitz S. 128 ff. mwN.

$12 \mathrm{Zu}$ diesen und anderen Einwänden Kübler, Effizienz als Rechtsprinzip, FS für Steindorff (1990) S. 687 ff. mwN.

13 Rittner JZ 2005, 668, 669; ähnlich Bydlinski (FN 3). 
engen Begriff der Rechtswissenschaft aus: Sie wird mit der professionellen Praxis dogmatischen Argumentierens und Begründens gleichgesetzt, die sich literarisch vorwiegend in den Kommentaren niederschlägt. Dabei wird offenbar übersehen, dass sich die wissenschaftliche Arbeit an rechtlichen Texten und Institutionen immer schon der Hilfe anderer Disziplinen bedient hat. Ursprünglich waren das vor allem Geschichte, Philologie und Philosophie; später sind die Sozialwissenschaften hinzugetreten. Sie kooperieren vorzugsweise mit bestimmten Rechtsgebieten: Die Kriminologie mit dem Strafrecht, Verwaltungs- und politische Wissenschaft mit dem öffentlichen Recht; Familiensoziologie mit dem Familienrecht. Das schließt die Wirtschaftswissenschaften nicht aus: Kartellrecht ließ sich von Wettbewerbstheorie, Bilanzrecht von Bilanztheorie und Steuerrecht von Finanzwissenschaft inspirieren, bevor von »law and economics« die Rede war.

Die Wirtschaftswissenschaften haben gelegentlich dazu beigetragen, die Ängste vor dem Verlust der Eigenständigkeit der Jurisprudenz zu schüren. In der Literatur zu Law and Economics werden traditionell drei Funktionen der ökonomischen Analyse unterschieden ${ }^{14}$. Sie ist »positiv«, d.h. operiert deskriptiv, wo sie bestehende Institute oder Regeln anhand ihrer ökonomischen Implikationen erklärt. Sie verfährt »präskriptiv«, d.h. rechtspolitisch, wo sie ökonomisch vorteilhafte Regelungen empfiehlt; derartige Ratschläge können an die Gesetzgebung, die Rechtsprechung oder die Vertragspraxis adressiert sein. Und sie argumentiert »normativ«, wo sie umfassende evolutionstheoretische Deutungen der Gesellschafts- und der Rechtsordnung entwirf ${ }^{15}$. Hier tritt die Empirie hinter die Spekulation zurück; Märkte sind der Regulierung allemal überlegen; und ein spezifisches Modell (das des vollständigen Markts) wird zum dominierenden Paradigma. Diese dritte Deutung aufnehmend hat Richard Posner den Aufstieg von Law and Economics mit dem »Decline of Law as an Autonomous Discipline « gleichgesetz ${ }^{16}$; an anderer Stelle hat er der traditionellen Rechtswissenschaft attestiert, dass sie sich in einem vorwissenschaftlichen Zustand befinde ${ }^{17}$.

Der andere grundlegende Einwand richtet sich gegen das Verhaltensmodell der neoklassischen Theorie. Es beruht auf der generellen Annahme rationalen Verhaltens der Marktteilnehmer: Sie maximieren ihren Vorteil auf der Basis stabiler Präferenzen und unter Nutzung der optimalen Menge an Information ${ }^{18}$. Gegen dieses Konstrukt des »homo oeconomicus ${ }^{19}{ }^{19}$ wird eingewandt, es reduziere das facettenreiche Menschenbild komplexer Rechtsordnungen zur linearen Funktion der Nutzen- oder Vorteilsmaximierung ${ }^{20}$. Diese Kritik vernachlässigt, dass es im rechtsökonomischen Zusammen-

14 Dazu Jolls/Sunstein/Thaler, A Behavioral Approach to Law and Economics, Stanford L. Rev. 50 (1997/98), 1471, 1474.

15 Rose-Ackermann, Recht und Ökonomie: Paradigma, Politik oder Philosophie, in: Ott/Schäfer (Hrsg.), Allokationseffizienz in der Rechtsordnung (1989), 269, $277 \mathrm{ff.}$

16 So der Titel eines Aufsatzes in Harvard L. Rev. 100 (1986/87), $761 \mathrm{ff}$.

17 Journal of Legal Studies 1 (1972), $437 \mathrm{ff}$.

18 Gute Darstellung bei Englerth, Behavioral Law and Economics - Eine kritische Einführung, Preprints of the Max Planck Institute for Research on Collective Goods, Bonn 2004/ 11, S. 4 ff. mwN.

19 Zu ihm eingehend Kirchgässner, Homo Oeconomicus (1991) insbesondere S. 12 ff. 
hang vor allem darum geht, die verhaltenssteuernde Funktion des Rechts zu erfassen; dafür dienen unterschiedliche Ausprägungen der $»$ rational choice theory $\ll^{21}$. Zudem verwendet die Rechtswissenschaft ähnliche Begriffe; auch sie hat es mit Menschen »als Käufer, Verkäufer, Konsument, Sparer, Importeur, Exporteur, Unternehmer, Händler, Angestellter, Arbeiter usf.« zu tun ${ }^{22}$. Das Verfasssungsrecht ist der ständigen Versuchung ausgesetzt, ein durch sozialethische Elemente angereichertes »Menschenbild des Grundgesetzes « zu entwerfen; es wird mit guten Gründen davor gewarnt, ein solches Konstrukt im Wege der Verfassungsinterpretation normativ festzuschreiben ${ }^{23}$.

Die Kritik am »rational choice«-Ansatz ist aber dort berechtigt, wo die Modellannahme der Rationalität für die Wirklichkeit ausgegeben und deshalb zwingende Rechtsregeln ohne Rücksicht auf empirische Erfahrungen und Befunde als »Paternalismus« verurteilt werden ${ }^{24}$. Diese Überinterpretation der Theorie des rationalen Verhaltens berührt sich mit der »präskriptiven « Funktion der Rechtsökonomie: Hier wie dort wird auf Empirie beruhende Analyse durch auf Annahmen gestützte Spekulation ersetzt.

Ein korrektes Verständnis des neoklassischen Modells hat nie einen Zweifel daran gelassen, dass sich Menschen im Einzelfall auch irrational verhalten können. Es wurde aber angenommen, dass diese Abweichungen vom Zufall bestimmt werden und sich deshalb auf die Dauer oder bei größerer Zahl ausgleichen ${ }^{25}$. Dass diese Annahme nicht unproblematisch ist, hat sich mit Hilfe von Laborexperimenten gezeigt (deren Anfänge bereits in den 30er und 40er Jahren des letzten Jahrhunderts liegen) ${ }^{26}$. Abweichungen vom Rationalverhalten werden seit Mitte der 70er Jahre des letzten Jahrhunderts systematisch erforscht. Die Psychologen Daniel Kahneman und Amos Tversky sowie eine Reihe von Ökonomen begannen, die von der kognitiven Psychologie entwickelten Laborexperimente auf ökonomische Fragestellungen anzuwenden ${ }^{27}$. Diese

20 Fezer, JZ 1986, 817, 822 und 824; Eder (FN 3) S. 12 und 15 f.

21 Eingehende Erörterung bei Korobkin/Ulen, Law and Behavioral Science: Removing the Rationality Assumption from Law and Economics, California L. Rev. 88 (2000), 1051, $1060 \mathrm{ff}$.

22 So - für die Wirtschaftswissenschaften - Häuser, Zum Menschenbild der Nationalökonomie, in: Duncker (Hrsg.), Beiträge zu einer aktuellen Anthropologie (2006), 285. Der Beitrag legt dar, dass die deutsche Historische Schule den Begriff des homo oeconomicus zurückgewiesen hat, aber nicht in der Lage war, dem »rational choice«-Ansatz eine gleichwertige Theorie entgegenzusetzen.

23 Stolleis, Das Menschenbild der Verfassung, in Duncker S. 369 ff.

24 Englerth (FN 18) S. 50 ff. mwN.

25 Dazu näher unter IV.

26 Einen guten historischen Überblick findet man bei Roth/Kagel, Handbook of Experimental Economics (1995), Princeton University Press, Chapter 1 (Roth). Siehe auch Simon mit seinem Begriff der »bounded rationality«; A Behavioral Model of Rational Choice, Quarterly Journal of Economics 69 (1955), 99 ff.

27 Bahnbrechend war beispielsweise ihre Arbeit »Prospect Theory: An Analysis of Decision Under Risk «, Econometrica 47 (1979), 263 ff. Für sie hat Kahneman (zusammen mit Vernon Smith) 2002 den Nobelpreis erhalten (Tversky war schon verstorben). Zum aktuellen Stand Guthrie, Prospect Theory, Risk Preference and the Law, Northwestern Univ. L. Rev. 97 (2003), $1116 \mathrm{ff}$. 
methodische Neuausrichtung lässt sich als die eigentliche Geburtsstunde von »behavioral economics « ansehen, die sich mittlerweile zu einem höchst lebendigen und expandierenden Zweig der Wirtschaftswissenschaften entwickelt hat.

\section{III.}

Definiert man das neoklassische Verhaltensmodell als rationales Entscheiden, das durch Eigennutzorientierung, konsistente Präferenzen und optimierendes Verhalten bestimmt wird, dann lassen sich wichtige Befunde der verhaltensökonomischen Forschung als systematische Relativierungen dieser Prämissen darstellen.

1. Für die Eigeninteressen, die der homo oeconomicus verfolgt, stellt sich die Frage ihrer Definition. Lässt man jede beliebige Erwartungsnutzenfunktion (expected utility) zu, dann verliert der Begriff seine Abgrenzungsfunktion: Eigensüchtig handeln der Märtyrer ebenso wie der Karrierist ${ }^{28}$. Deshalb bevorzugt die Rechtsökonomie eine engere (oder »dickere «) Fassung des Begriffs: Das handelnde Individuum wird durch »self-interest«, durch das Ziel der Mehrung des eigenen Nutzens, insbesondere des eigenen Wohlstands motiviert ${ }^{29}$. Die faktische Schwäche dieser Annahme wird durch ein mittlerweile berühmtes spieltheoretisches Experiment, das »Ultimatumspiel«, illustriert $^{30}$. Die Grundform findet zwischen zwei einander unbekannten Spielern statt. A wird aufgefordert, B einen Vorschlag über die Aufteilung eines dafür gewährten Geldbetrages, etwa von 100 N,- zu unterbreiten. Nimmt B an, dann wird entsprechend geteilt. Lehnt B ab, dann gehen beide leer aus.

Geht man von den Annahmen der neoklassischen Theorie aus, dann ist eine extrem ungleiche Verteilung zu erwarten: A kann seinen Nutzen maximieren, indem er B nur einen Euro anbietet und den Rest für sich behält, denn B wird sich finanziell immer noch besser stellen, wenn er diesen Minimalbetrag annimmt und nicht ablehnt. Diese Erwartung wird durch das - mittlerweile in vielen modifizierten Versionen wiederholte - Spiel nicht bestätigt: Im Durchschnitt wird eine Teilung nicht von $99: 1$, sondern von 63 : 37 vorgeschlagen; das häufigste Angebot zielt auf hälftige Teilung; und Angebote von weniger als $20 \%$ werden fast immer abgelehnt ${ }^{31}$. Diese Zahlen ändern sich, wenn die Randbedingungen modifiziert werden: Der B angebotene Teil sinkt, wenn A der Eindruck vermittelt wird, Anspruch auf einen größeren Prozentsatz zu haben oder wenn es sich bei den Spielern um Studierende der Wirtschaftswissenschaften handelt, die mit der neoklassischen Theorie vertraut sind und sich dem »rational choice «-Ansatz verpflichtet fühlen ${ }^{32}$.

28 Englerth (FN 18) S. 8.

29 Korobkin/Ulen (FN 21) S. 1064 ff.

30 Es geht auf Güth/Schmittberger/Schwarze, An Experimental Analysis of Ultimatum Bargaining, Journal of Economic Behavior and Organisation 3 (1982) 367 ff. zurück; aktualisierte Darstellungen bei Camerer, Behavioral Game Theory (2003), Princeton: Princeton University Press, Jolls/Sunstein/Thaler (FN 14), 1489 ff.; Englerth (FN 18) 10 ff.

31 Korobkin/Ulen (FN 21) S. 1135 ff.

32 Camerer (FN 30) und Arlen, The Future of Behavioral Economics of Law, Vanderbilt L Rev. 51 (1998), 1765, 1785 ff. 
Die Ergebnisse des Ultimatumspiels werden weithin übereinstimmend damit erklärt, dass die Menschen einer sozialen Regel der Fairness folgen ${ }^{33}$. Deshalb sind sie bereit, durch faire Vorschläge auf einen Teil des erreichbaren Vorteils zu verzichten, bzw. Nachteile in Kauf zu nehmen, um das unfaire Verhalten anderer zu bestrafen ${ }^{34}$. Die Fairnesstheorien werden durch Beobachtungen im Alltag bestätigt: Leistungen werden auch dort entgolten, wo die Zahlung in eine (angekettete oder fest verankerte) Sammelbüchse erfolgt und ihr Eingang nicht kontrolliert wird; oder: potentielle Käufer sind bereit, besonders preiswerte Waren zu boykottieren, um auf diese Weise der Ausbeutung von Kinderarbeit entgegenzuwirken ${ }^{35}$. Weniger eindeutig ist, welche Faktoren den Inhalt dessen bestimmen, was (kollektiv) als fair betrachtet wird. Für das Ultimatumspiel wird angenommen, dass es für die Beteiligten eine »Referenztransaktion « gibt, deren Konditionen als fair gelten; dann ist »unfair«, was zu weit von diesem Maßstab abweicht ${ }^{36}$. Zugleich ist evident, dass der Fairness-Standard auch von Eigeninteressen bestimmt wird; das wird als »self-serving bias « bezeichnet ${ }^{37}$.

2. Zu den stabilen Präferenzen des »rational choice«-Ansatzes gehört auch eine konsistente Einschätzung von Risiken: Marktakteure können risikoscheu oder risikofreudig sein; aber sie sind immer das eine oder das andere. Diese Annahme wird durch die »Prospect Theory ${ }^{38}$ erschüttert. Zu ihren Grundlagen zählt ein Experiment, bei dem den Beteiligten die Auswahl zwischen einer sicheren Zahlung von \$240 und der 25\%igen Chance angeboten wurde, \$1.000 zu gewinnen; hier wählten $84 \%$ die sichere Zahlung, obwohl ihr Wert geringfügig hinter dem Erwartungswert der unsicheren Zahlung zurückbleibt. Wurde ihnen die Wahl zwischen einem sicheren Verlust von \$ 750 und einem 75\%-igen Risiko eröffnet, \$1.000 zu verlieren, wählten $87 \%$ die riskante Alternative, obwohl beide Optionen denselben Erwartungswert haben. Der - mit anderen Anordnungen häufig wiederholte - Versuch zeigt ein verbreitetes Handlungsmuster: Wo es um die Mehrung des Vermögens, um Zugewinn geht, sind wir risikoscheu: Das sichere Plus wird der Lotterie mit dem höheren Erwartungswert vorgezogen. Wo es hingegen um die Verhinderung oder Minimierung von Verlusten geht, sind wir risikogeneigt: Um die sichere Einbuße zu meiden, sind wir bereit, die Gefahren weit höherer Verluste in Kauf zu nehmen ${ }^{39}$.

33 Theoretische Ansätze für die Modellierung sozialer Präferenzen finden sich vor allem bei Fehr/Schmidt, A Theory of Fairness, Competition and Co-operation, Quarterly Journal of Economics 114 (1999), 817 ff., bei Bolton/Ockenfels, ERC: A Theory of Equity, Reciprocity and Competition, American Economic Review, 90 (2000), 166 ff. und bei Rabin, Incorporating Fairness into Game Theory and Economics, American Economic Review 83 (1993), $1281 \mathrm{ff}$.

34 Siehe dazu Kosfeld/Riedl in diesem Heft S. 139 ff.

$35 \mathrm{Zu}$ diesen Beispielen Jolls/Sunstein/Thaler (FN 14), 1493 ff.

36 Jolls/Sunstein/Thaler S. 1496.

37 Für das Ultimatumspiel siehe Kagel/Kim/Moser, Fairness in Ultimatum Games with Asymmetric Information and Asymmetric Payoffs. Games and Economic Behavior 13, S. $100 \mathrm{ff}$. und allgemein Englerth (FN 18) S. 14 f. mwN.

38 Kahneman/Tversky (FN 27); Guthrie (FN 27).

39 Korobkin/Ulen (FN 21) S. 1104 ff. 
Noch genauer: Die Risikobereitschaft wird dadurch bestimmt, was als Gewinn und was als Verlust betrachtet wird; das wird als »frame« (Rahmen) bezeichnet. Wie die Risikobereitschaft Entscheidungen im Bereich von Zivilprozessen beeinflusst, ist durch mehrere Experimente ermittelt worden, in denen es um die Beendigung des Verfahrens durch Vergleich ging ${ }^{40}$. Sie zeigten durchweg, dass die Vergleichsbereitschaft bei gleichem Prozessrisiko sehr viel größer war, wo die dem Kläger angebotene Summe seinen Verlust überstieg ${ }^{41}$, und sehr viel geringer, wo die Einbußen durch den Vergleich nicht mehr vollständig ausgeglichen wurden ${ }^{42}$. Ein weiteres Anwendungsfeld der Prospekttheorie sind Haftungsregeln, deren Optimalität von der spezifschen Form der Risikopräferenzen abhängt ${ }^{43}$. Schließlich gibt es Fälle, in denen die Wahrnehmung von Gewinnchancen und Verlustrisiken von außen, etwa durch die Ausgestaltung einer Transaktion, beeinflusst werden kann. Das eröffnet die Möglichkeit der Manipulation, der das Recht - Gesetzgebung oder Judikatur - durch Informationspflichten und Transparenzgebote entgegenwirken kann.

3. Die Annahme, dass die am Güteraustausch beteiligten Subjekte ihren Nutzen systematisch verfolgen und maximieren, konfrontiert schon die neoklassische Ökonomie mit Problemen: Es ist evident, dass vielfach ohne genaue Information über die zu erwartenden Folgen entschieden wird ${ }^{44}$. Trotzdem ist kein Bruch mit den Modellprämissen zu verzeichnen: Es ist durchaus rational, auf Information zu verzichten, wenn die Kosten ihrer Gewinnung und Verwertung höher sind als der von ihrem Gebrauch zu erwartende Nutzen ${ }^{45}$. Was bei großen Transaktionen als informationsökonomisches Kalkül expliziert wird, geht als intuitives Abwägen in unsere Alltagsgeschäfte ein: Wir decken meist unseren Bedarf an Brot und Obst ohne vorherigen Preisvergleich, da er uns zu viel Zeit abnötigte. Es sind aber wiederum Einsichten der kognitiven Psychologie, die das Profil des homo oeconomicus verändern:

$\mathrm{Zu}$ den nachgewiesenen Befunden zählt der »confirmatory bias«, der die Aufnahme und Verarbeitung von Nachrichten - etwa bei der Lektüre der Tageszeitung - bestimmt: Informationen werden wahrgenommen, wenn sie den Überzeugungen des Lesers entsprechen; sie werden übersehen oder übergangen, wo dies nicht der Fall ist ${ }^{46}$. Derartige Verzerrungen werden als »bias« bezeichnet. Besonders gut dokumentiert ist der »overconfidence bias«, der sich vor allem in der Überschätzung der eigenen Fähig-

40 Korobkin/Guthrie, Opening Offers and Out of Court Settlement: A Little Moderation May Not Go a Long Way, Ohio State Journal of Dispute Resolution 10 (1994), 1 ff.; dies., Psychological Barriers to Litigation Settlement: An Experimental Approach, Michigan L. Rev. 93 (1994), $107 \mathrm{ff}$.

41 Das amerikanische Haftpflichtrecht gewährt in vielen Fällen »general « und/oder »punitive damages«, deren - häufig von der Jury festgesetzter - Betrag den Umfang des erlittenen materiellen Schadens erheblich übersteigen kann.

42 Korobkin/Ulen (FN 21) S. $1106 \mathrm{f}$.

43 Dazu Eide sowie Bigus in diesem Heft S. 159 ff. und S. 194 ff.

44 Englerth (FN 18) S. 26 ff.

45 Stigler, The Economics of Information, Journal of Political Science 69 (1961), 213ff.; Kirchgässner (FN 19), S. 71.

46 Rabin/Schrag, First Impressions Matter: A Model of Confirmatory Bias. Quarterly Journal of Economics (1999), 114, S.37 ff. 
keiten manifestiert: $90 \%$ aller Autofahrer glauben, dass sie überdurchschnittlich gute und sichere Verkehrsteilnehmer sind und deshalb weniger häufig in schwere Unfälle verwickelt werden ${ }^{47}$; und $94 \%$ der Universitätsprofessoren halten sich für ihren Kollegen überlegen ${ }^{48}$. Eine vergleichbare Verzerrung ist der »hindsight bias«, der von der ex post- auf die ex ante-Wahrnehmung schließt. Die Kenntnis der Unfallursachen und des Schadenverlaufes festigt den Glauben daran, diese Ereignisse seien voraussehbar gewesen. Das kann sich im Haftpflichtprozess vor allem dort zum Nachteil des Beklagten auswirken, wo die Entscheidung von der Jury, d.h. von Laienrichtern getroffen wird $^{49}$. Ein weiteres Beispiel ist der »just world bias«, der dazu verführt, das Schicksal von Verbrechens- oder Diskriminierungsopfern für wohlverdient zu halten ${ }^{50}$. Diese Befunde konfrontieren mit der Frage, inwieweit derartige Verzerrungen mittels Recht eliminiert oder minimiert werden können ${ }^{51}$.

Weitere Einschränkungen der Rationalitätsannahme resultieren aus den kognitiven Vereinfachungsverfahren, die als Heuristiken bezeichnet werden. Es entspricht den Grundsätzen der Informationsökonomie (insbesondere der Bayes-Regel), dass Entscheidungen auf durch Erfahrung gewonnenes Hintergrundwissen, die »base rate«, gestützt werden, die durch neue Erfahrungen aktualisiert wird. Dabei kommt es indessen regelmäßig zu Verzerrungen. Die Wahrscheinlichkeit bestimmter Ereignisse wird überbewertet, weil sie besonders aktuell oder besonders einprägsam sind oder weil sie repräsentativ erscheinen ${ }^{52}$. In einem (amerikanischen) Experiment sollten die Teilnehmer schätzen, wie viele Worte eines Textes auf »ing « endeten und in wie vielen Worten $» n \ll$ der zweitletzte Buchstabe war. Da »n« in »ing « enthalten ist, tritt dieser Fall häufiger auf. Trotzdem hielten die meisten Teilnehmer »ing « für häufiger, weil ihnen diese Endung viel geläufiger war ${ }^{53}$.

IV.

Die vorstehend nur grob umrissenen Ansätze sind nicht nur bei Ökonomen sondern auch bei Juristen auf lebhafte Kritik gestoßen, die die Aussagekraft und Zuverlässigkeit der Laborbefunde bezweifeln ${ }^{54}$. Hier ist gewiss zu differenzieren. Einem einmali-

47 Taylor, Positive Illusions (1989) S. 10 ff.

48 Englerth (FN 18) S. 31.

49 Kamin/Rachlinsky, Ex Post ? Ex Ante, Law \& Human Behavior 19 (1995), 89 ff.; Labine/ Labine, Determination of Negligence and the Hindsight Bias, Law \& Human Behavior 20 (1996), $50 \mathrm{ff}$.

50 Dazu Dharmapala/Garoupa/McAdams in diesem Heft S. 118 ff.

51 Dazu Jolls/Sunstein, Debiasing by Law, Journal of Legal Studies 35 (2006), 199 ff.; und Deffains/Ferey in diesem Heft S. 174 ff.

52 Das wird als »availability heuristic « und »representativeness heuristic « bezeichnet; grundlegend Kahneman/Slovic/Tversky, Judgment Under Uncertainty: Heuristics and Biases (1982).

53 Kahneman/Tversky, Extensional versus Intuitive Reasoning: The Conjunction Fallacy in Probability Judgment, Psychological Review 90 (1983), 295 ff.

54 Posner, Rational Choice, Behavioral Economics, and the Law, Stanford L. Rev. 50 (1998), 1551, 1570; Arlen (FN 31), $1768 \mathrm{f}$. 
gen Experiment mit einer kleinen Gruppe von Studierenden werden in der Regel keine gesicherten Einsichten zu entnehmen sein; das Bild ändert sich jedoch, wenn unterschiedliche Versuchsanordnungen in unterschiedlichen Institutionen und Ländern regelmäßig sich weitgehend deckende Befunde ergeben; und das trifft auf die zu III. referierten Ergebnisse $\mathrm{zu}^{55}$. Eine empirische Wirtschaftsforschung, die sich ausschließlich in der Realität gewonnener Daten bedient, wird Ergebnisse mit hoher externer Validität vermitteln; aber dieser Ansatz verlangt einen beträchtlichen Aufwand und ist aufgrund der Datenlage vielfach nicht in der Lage, die die Verhaltensökonomie beschäftigenden Fragen zu beantworten.

Zugleich wird von juristischer Seite dem Ansatz wegen seines rein empirischen Charakters das wirtschaftswissenschaftliche Heimatrecht abgesprochen. Er sei »antitheoretical« und »undertheorized «; wenn er überhaupt auf einer Theorie beruhe, sei dies keine ökonomische Theorie ${ }^{56}$. Außerdem wird seine Relevanz verneint: Die Abweichungen vom »rational choice«-Modell seien bloß zufällig und nicht systematisch $^{57}$. Das ist zumindest missverständlich. Behavioral Law and Economics ist weiterhin der Ökonomie verhaftet: Es werden die Bedingungen individuellen Handelns untersucht, die allen Menschen gemeinsam sind und nicht auf Besonderheiten der Schichtzugehörigkeit oder des Bildungsstandes beruhen ${ }^{58}$.

Das soll an einem Beispiel verdeutlicht werden: Für drei Jahrzehnte zählte die Hypothese der Kapitalmarkteffizienz ${ }^{59} \mathrm{zu}$ den Fundamenten der Finanzierungstheorie. Sie beruhte auf drei Annahmen, die als weithin gesichert galten: Investoren verhalten sich grundsätzlich rational; wo dies im Einzelfall nicht zutrifft, beruhen die Abweichungen auf Zufall und gleichen sich aus; etwa noch verbleibende Abweichungen werden durch die Arbitrage des Berufshandels ausgeglichen ${ }^{60}$. Deshalb war davon auszugehen, dass die sich selber überlassenen Finanzmärkte die optimale Allokation der verfügbaren Ersparnisse bewirken. Mittlerweile sind die Annahmen, auf denen die Effizienzhypothese beruht, erschüttert ${ }^{61}$. Die erwähnten Befunde etwa der Prospect Theory oder der Heuristiken vermitteln die Einsicht, dass das Verhalten der Anleger nicht nur sporadisch, sondern systematisch vom Modell des rationalen Entscheidens abweicht und dass diese Divergenzen nicht mehr durch den Markt ausgeglichen wer-

55 Zur Frage der externen Validität von Experimenten, am Beispiel von Experimenten über Korruption, siehe auch Ortmann in diesem Heft S. $103 \mathrm{ff}$.

56 Posner S. 1552, 1558 und 1559

57 Arlen S. 1766.

58 Einleuchtend Englerth S. $33 \mathrm{ff}$.

59 Die Efficient Capital Market Hypothesis (ECMH) wird in drei Versionen vertreten. Die schwächste Form besagt, dass der Marktverlauf »random walk « entspricht und deshalb aus den Kursverläufen der Vergangenheit keine Schlüsse auf die künftige Entwicklung gezogen werden können. Die stärkste Form besagt, dass jede verfügbare Information sofort in den Kurs eingeht (dann lässt sich Insiderwissen nicht gewinnbringend verwerten). Die vermittelnde Ansicht hält dafür, dass dies nur für veröffentlichte Informationen gilt (dann sind Insidergeschäfte möglich).

60 Fama, Efficient Capital Markets: A Review of Theory and Empirical Work, Journal of Finance 25 (1970), $283 \mathrm{ff}$.

61 Shleifer, Inefficient Markets. An Introduction to Behavioral Finance (2000) S. $10 \mathrm{ff}$. 
den; damit erweist sich auch die Arbitrage als ein riskantes Geschäft, dessen Wirksamkeit überschätzt worden war. Daraus folgt, dass die Hypothese der Kapitalmarkteffizienz fragwürdig geworden ist. Das hat erhebliche rechtspolitische Implikationen. $\mathrm{Zu}$ ihnen zählt etwa die Frage, inwieweit staatliche Systeme der Sozialversicherung zu Gunsten von Modellen privaten Sparens und Investierens zurückgedrängt werden sollten: Wenn die private Investitionstätigkeit in erheblichem Maße durch Irrationalität bestimmt wird, dürfte es sich empfehlen, ein vom Staat beaufsichtigtes System der Social Security zumindest als Sockel der Altersversorgung beizubehalten ${ }^{62}$.

Damit lässt sich abschließend festhalten, dass die auf Experimente gestützte Einbeziehung von Einsichten der kognitiven Psychologie nicht bedeutet, dass das Standardmodell von Law and Economics aufgegeben würde. Der verhaltensökonomische Ansatz verspricht vielmehr konkrete Verbesserungen dieses Modells: Seine Prämissen werden der Realität angenähert; damit wächst die Plausibilität der ökonomischen Erklärung rechtlicher Institutionen; und sie dürfte wiederum die Zuverlässigkeit der für die Rechtspolitik erheblichen Prognosen der Folgen von Gesetzen, Urteilen und Transaktionen erhöhen ${ }^{63}$. Geschwächt wird allein die Persuasivität des »normativen « oder evolutionstheoretischen Verständnisses der Rechtsökonomie, das das neoklassische Modell in ein politisches Ordnungskonzept transformieren möchte ${ }^{64}$. Auch das ist zu begrüßen: Es kann die Zusammenarbeit von Ökonomen und Juristen nur fördern, wenn sie sich der sozialwissenschaftlichen Bezüge ihrer Disziplinen bewusst sind und bleiben; dazu möchte dieses Heft einen bescheidenen Beitrag leisten.

62 Shleifer S. $184 \mathrm{ff}$.

63 Dazu einleuchtend Jolls/Sunstein/Thaler (FN 14), S. $1545 \mathrm{ff}$.

64 Dieser Effekt der Verhaltensökonomie trägt zur negativen Einschätzung dieser Forschungsrichtung durch Posner bei; vgl. oben FN 54. 